\title{
Novel Electroluminescent Polymer Derived from Pyrene-Functionalized Polyaniline
}

\author{
Chellachamy Anbalagan Amarnath, Hyoung Kun Kim, ${ }^{\ddagger}$ Dong Kee Yi, ${ }^{\dagger, *}$ Sanghyup Lee, ${ }^{\S}$ Young Rag Do, ${ }^{*}$ and Ungyu Paik \\ Department of Energy Engineering, Hanyang University, Seoul 133-791, Korea \\ E-mail: upaik@hanyang.ac.kr \\ ${ }^{\dagger}$ Gachon Bionano Research Institute, Department of BioNano Technology, Kyungwon University, Seoul 461-701, Korea \\ EE-mail: vitalis@kyungwon.ac.kr \\ ¿Department of Chemistry, Kookmin University, Seoul 136-701, Korea \\ ${ }^{\S}$ Water Research Center, KIST, Seoul 130-650, Korea \\ Received November 23, 2010, Accepted March 2, 2011
}

\begin{abstract}
A solution processable polymer was synthesized, by incorporating pyrene groups into the backbone of the polyaniline chain, and used as an emissive layer in an organic light emitting diode. The polyaniline base was reacted with acid chloride of pyrene butyric acid to form pyrene-functionalized polyaniline chains. The source of pyrene moiety was acid chloride of pyrene butyric acid. The formation of polymer from acid chloride of pyrene butyric acid and polyaniline was confirmed by the FTIR and ${ }^{1} \mathrm{H}-\mathrm{NMR}$ spectroscopy. Differential scanning calorimetry revealed high glass transition temperature of $210{ }^{\circ} \mathrm{C}$. Due to the presence of pyrene moieties in the backbone, the polyaniline synthesized in the present study is solution processable with light emitting property. The photoluminescence spectrum of the polymer revealed that emission lies in the blue region, with a peak at $475 \mathrm{~nm}$. The light emitting device of this polymer exhibits the turn-on voltage of $15 \mathrm{~V}$.
\end{abstract}

Key Words : Polyaniline, Electroluminescent, Pyrene group, $N$-Alkylation

\section{Introduction}

The field of conducting polymers has recently focused on the study of polyaniline because of its unique electro-optical properties and market potential. ${ }^{1}$ However, as is common with other conjugated polymers, the application of polyaniline (PANI) is limited by its poor melt and solution processability. Improved solubility of PANI can be achieved by introducing various alkyl and alkoxy substituents to the polymer backbone., ${ }^{2,3}$ Therefore, alkyl- and alkoxy-substituted PANI have been intensively studied. On the other hand, there are little reports on the pyrene functionalized PANI.

Organic electroluminescent (EL) devices are of growing interest in display applications because they are able to emit colors throughout the visible spectrum and with high luminous efficiency. There have been extensive studies on using low molecular weight complexes to make organic EL devices in order to achieve high brightness, multicolor emission, improved durability, and desired efficiency. ${ }^{4-18}$ From a materials perspective, organic light emitting diodes (OLEDs) are primarily prepared from either small molecules or polymers. ${ }^{19-22}$ Small molecules are advantageous in that they can be highly purified and vacuum deposited in multilayer stacks, both important for display lifetime and efficiency. However, vacuum deposition techniques require expensive capital equipment, a limitation to practical display size, and have significant problems in achieving full color displays at high volume using masking technologies. ${ }^{23}$ Polymers are generally of lower purity than small molecules but can access larger display sizes and full color at much lower costs via solution-based deposition techniques. ${ }^{24,25}$ Our interest is in the family of materials that combines the advantages of both small molecule and polymer approaches to OLEDs specifically-polyaniline and pyrene based hybrids. In this work we have functionalized PANI backbone with the pyrene group and we utilized the pyrene-functionalized polyaniline as novel light emitting material. Generally, many researchers have utilized PANI in light emitting diodes as hole injection layer and anode. However, we used polyaniline as a light emitting electroluminescent material for the first time in the light emitting diode. Furthermore, this pyrene-functionalized PANI offers many advantages for OLEDs including ease synthesis, low cost, high glass-transition temperature and high solubility.

\section{Experimental}

Ammonium persulfate, pyrene butyric acid, $m$-cresol, thionyl chloride were purchased from Sigma Aldrich, Seoul, Korea and used as purchased. Aniline from Sigma Aldrich was vacuum distilled prior to use. The solvents like $m$ cresol, $\mathrm{N}, \mathrm{N}$-dimethylformamide, dimethyl sulfoxide, $\mathrm{N}$ methyl pyrrolidone, chloroform, dichloromethane, methanol were of analytical grade and were purchased from Sigma Aldrich, Seoul, Korea.

PANI salt was prepared by aqueous polymerization pathway by reported procedure. ${ }^{26}$ In a $1 \mathrm{~L}$ round-bottomed flask, $250 \mathrm{~mL}$ of $1 \mathrm{M} \mathrm{HCl}$ was taken and $5 \mathrm{~mL}$ of aniline was added slowly with stirring. To this mixture, $250 \mathrm{~mL}$ of $1 \mathrm{M}$ $\mathrm{HCl}$ containing ammonium persulfate $(11.2 \mathrm{~g})$ was added dropwise for 15-20 min and the solution was kept under constant stirring at $5-10{ }^{\circ} \mathrm{C}$. The reaction was allowed to 
continue for $4 \mathrm{~h}$ at $5-10{ }^{\circ} \mathrm{C}$. The precipitated PANI powder was filtered and washed with $2 \mathrm{~L}$ distilled water followed by $200 \mathrm{~mL}$ acetone. The PANI powder was dried at $80^{\circ} \mathrm{C}$ till a constant weight was reached.

PANI salt powder $(1.0 \mathrm{~g})$ synthesized above was stirred in $100 \mathrm{~mL}$ aqueous sodium hydroxide solution $(1.0 \mathrm{M})$ for $8 \mathrm{~h}$ at ambient temperature. PANI base powder was filtered, washed with excess water and finally with acetone and dried at $100{ }^{\circ} \mathrm{C}$ till a constant weight was reached.

An amount of $0.6 \mathrm{~g}(8 \mathrm{mmol})$ pyrene butyric acid was converted into its acid chloride by thionyl chloride ( $0.3 \mathrm{~g}, 10$ mmol). The freshly prepared acid chloride was made to react with the PANI base $(2 \mathrm{mmol})$ in $m$-cresol solvent. $N$ Acylation reaction was carried out in $120{ }^{\circ} \mathrm{C}$ for $12 \mathrm{~h}$. After the reaction time pyrene functionalized PANI solution in $\mathrm{m}-$ cresol was filtered in $0.25 \mu \mathrm{m}$ filter paper to remove the unreacted or agglomerated PANI particle. Thus obtained pyrene-functionalized PANI solution in $m$-cresol was dispersed in chloroform solvent to fabricate the device.

Fourier transform infrared spectrometer (FT-IR) analysis in the region of $400-3800 \mathrm{~cm}^{-1}$ was performed on potassium bromide pellets with a Nicol 760 MAGNA-IR spectrometer. ${ }^{1} \mathrm{H}-\mathrm{NMR}$ spectra were recorded in $300 \mathrm{MHz}{ }^{1} \mathrm{H}-\mathrm{NMR}$ (Bruker) spectrometer using DMSO- $d_{6}$ solvent containing tetramethylsilane (TMS) as an internal standard. The thermal property of the polymer sample was studied using a Perkin-Elmer DSC-7 with a heating and cooling rate of $10{ }^{\circ} \mathrm{C} \mathrm{min}-1$. The UV-vis absorption spectra are recorded using a Perkin-Elmer (Lambda 6) spectrophotometer. Absorption spectrum of dilute solution was recorded in the range 1100-300 nm using a pair of matched $3 \mathrm{~mL}$ stoppered quartz cells of $10 \mathrm{~mm}$ path length. The optical property was measured at room temperature by photoluminescence (PL) spectroscopy using a spectrophotometer (PSI co. Ltd. Darsar). Electroluminescence (EL) spectra for the pyrene-functionalized PANI sample were recorded with spectrophotometer (2035, Mac. pherson). The corresponding current-voltage characteristics were measured using a source meter (Keithley 2400) and colorimeter (Topcon BM-7).

\section{Results and Discussion}

Polyanilines are particularly attractive based on their easy preparation, excellent electrical properties, number of intrinsic redox states, possible processability and stability. The structure of PANI is known as a para-linked phenylene amineimine. The base form of polyaniline can, in principle, be described by the following general formula (Scheme 1):

In the generalized base form, $(1-\mathrm{x})$ measures the function of oxidized units. When $(1-\mathrm{x})=0$, the polymer has no such oxidized groups and is commonly known as a leucoemeraldine base. The fully oxidized form, $(1-\mathrm{x})=1$ is referred to as a pernigraniline base. The half-oxidized polymer, where the number of reduced units and oxidized units are equal, i.e. $(1-\mathrm{x})=0.5$, is of special importance and is termed as the emeraldine oxidation state or the emeraldine base. Partially oxidized emeraldine base is shown to be an alternating copolymer of reduced and oxidized repeat units. The value of $\mathrm{x}$ varies from 0 to 1 , but the percentage of carbon, hydrogen and nitrogen will be almost the same.

In the present study, the conventional PANI salt was synthesized and dedopped into PANI base via conventional synthesis procedure (Scheme 2). The PANI base was dissolved in $m$-cresol solvent and made to react with acid chloride of pyrene butyric acid at $120{ }^{\circ} \mathrm{C}$ for $12 \mathrm{~h}$ to form pyrene-functionalized PANI chains. Amine groups in the PANI chains were functionalized by butanoyl pyrene groups. In short, $50 \%$ of the aniline repeating units in the PANI chains were functionalized by butanoyl groups. The molecular amount of butanoyl pyrene group in the PANI group is 0.5:1. The formed PANI was precipitated with methanol, and characterized with FTIR and ${ }^{1} \mathrm{H}-\mathrm{NMR}$ spectroscopy.

FTIR and ${ }^{1} \mathrm{H}-\mathrm{NMR}$ proved the molecular structure of pyrene functionalized PANI. As representative systems, the

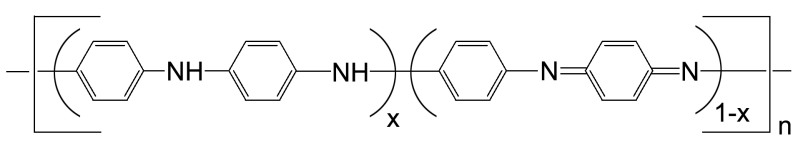

Scheme 1. General formula of PANI.
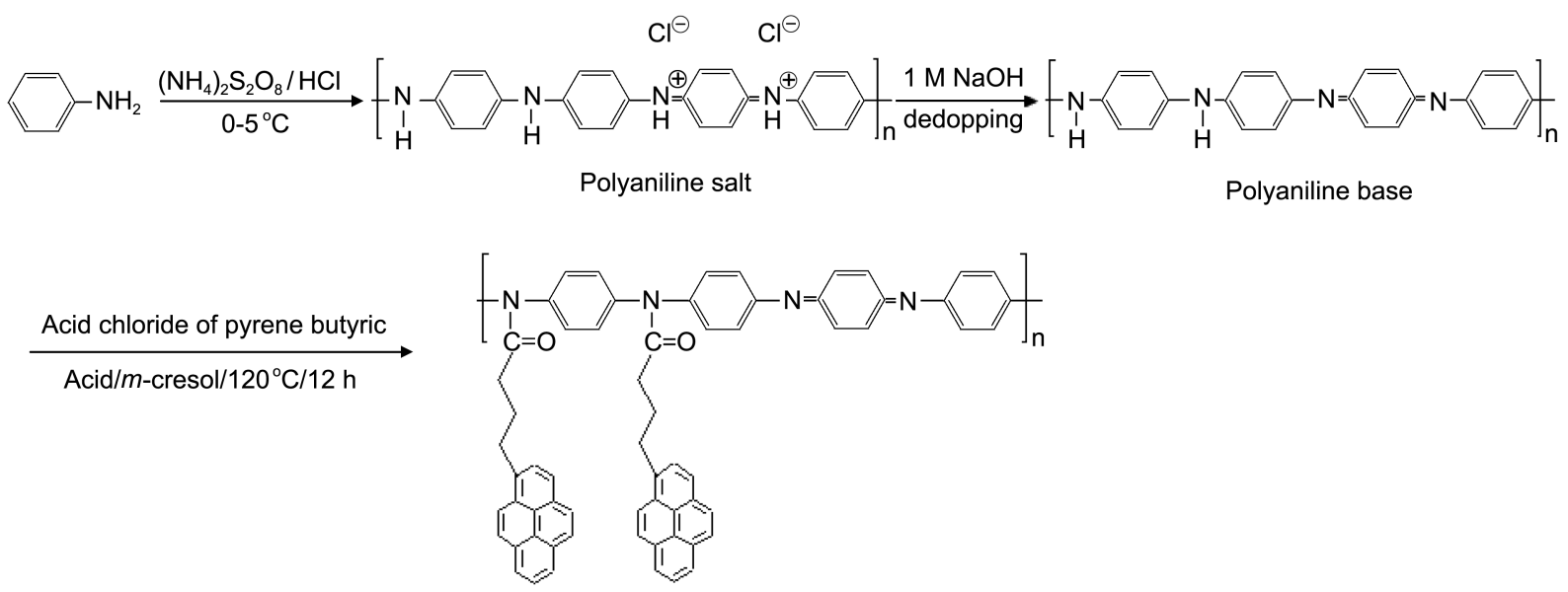

Pyrene functionalized polyaniline

Scheme 2. Synthesis of pyrene functionalized PANI. 


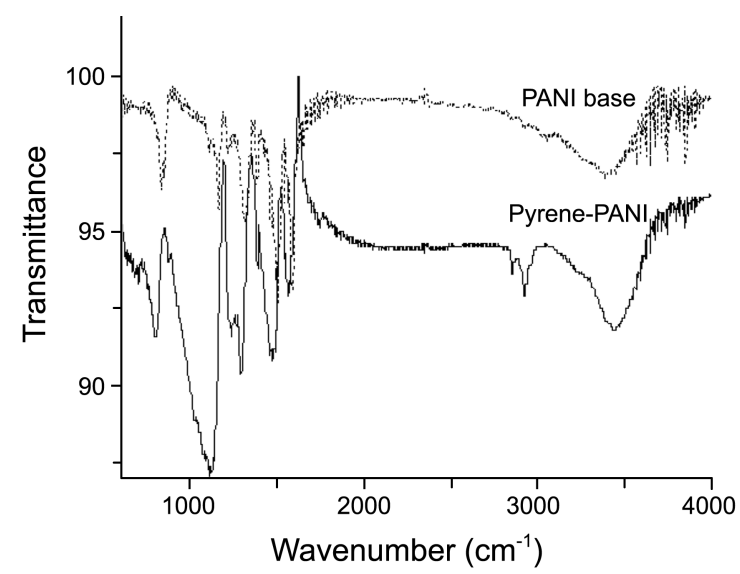

Figure 1. FTIR spectra of PANI base and pyrene functionalized PANI.

FTIR spectra of pyrene-functionalized PANI and PANI base are given in Figure 1. A similar spectral behavior was observed for PANI base and pyrene-functionalized PANI, except for the peak corresponding to $\mathrm{C}-\mathrm{H}$ stretching vibrations in pyrene containing PANI. The presence of bands at 2940 and $2850 \mathrm{~cm}^{-1}$ for pyrene-functionalized PANI are assigned to asymmetric and symmetric aliphatic $\mathrm{C}-\mathrm{H}$ stretching vibrations, respectively, ${ }^{27}$ indicating the existence of the alkyl substituents in the PANI chain. This peak proves the presence of butanoyl group (from acid chloride of pyrene butyric acid) in the PANI system. These peaks are the direct evidence for the presence of substituted pyrene moieties in the PANI chain. The ring stretching of quinonoid and benzenoid form in PANI samples are observed at 1570 and $1475 \mathrm{~cm}^{-1}$, respectively. The $\mathrm{C}-\mathrm{N}$ stretching band of an aromatic amine appears at $1300 \mathrm{~cm}^{-1}$. In the region of 1020$1170 \mathrm{~cm}^{-1}$ aromatic $\mathrm{C}-\mathrm{H}$ inplane-bending modes are usually observed. For PANI, a strong band characteristically appears at $1115 \mathrm{~cm}^{-1}$, which has been explained as an electronic band or a vibrational band of nitrogen quinone. A similar infrared behavior was observed for PANI salt reported in the literature. ${ }^{28}$ The peak for $\mathrm{C}=\mathrm{O}$ from butanoyl pyrene group in the PANI chain merges with the ring stretching peak of benzenoid, which appears at $1570 \mathrm{~cm}^{-1}$.

The ${ }^{1} \mathrm{H}-\mathrm{NMR}$ spectrum of the pyrene-functionalized PANI sample was recorded by dissolving the sample in DMSO- $d_{6}$ solvent. The ${ }^{1} \mathrm{H}-\mathrm{NMR}$ spectrum of pyrene functionalized PANI chains exhibits peaks near $\delta 7.2 \mathrm{ppm}$ due to the aromatic protons of the quinoid ring and the peaks at $\delta 6.9$ ppm due to the benzenoid protons. ${ }^{29}$ The peaks at $\delta 7.9$ to $8.5 \mathrm{ppm}$ in the spectrum of the pyrene functionalized polyaniline arise from the pyrene moieties in the main backbone of polyaniline chain.

The electronic absorption spectrum of polyaniline was recorded by diluting the pyrene functionalized polyaniline in chloroform solvent. The spectrum showed three peaks at around, $850 \mathrm{~nm}$ (polaron to $\pi$ ), $430 \mathrm{~nm}$ (polaron to $\pi^{*}$ ), and $340 \mathrm{~nm}\left(\pi-\pi^{*}\right)$. This is in accordance with the earlier report of Rao et al. ${ }^{29}$ This result supports the formation of pyrene functionalized polyaniline in the form of salt. During $N$ -

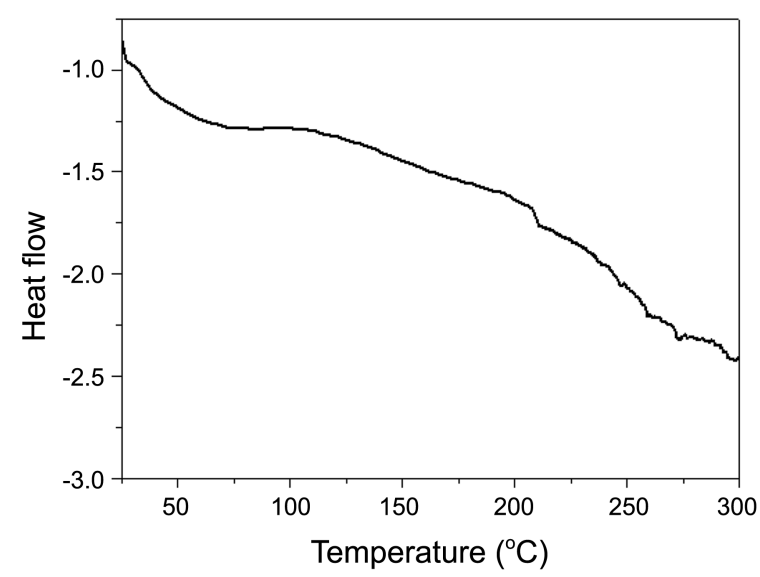

Figure 2. DSC thermogram of pyrene functionalized PANI.

acylation in the polyaniline backbone, the hydrochloride gas was obtained as a by-product of the reaction. The evolved by-product doped on the polyaniline chains and made pyrene functionalized polyaniline in the emeraldine salt form.

The differential scanning calorimetry (DSC) thermogram of pyrene functionalized PANI is shown in Figure 2 . It reveals high glass transition temperature $(T \mathrm{~g})$ of $210^{\circ} \mathrm{C}$ of the pyrene functionalized PANI and it is similar to the value of pyrene functionalized octavinylsilsesquioxane. ${ }^{30}$

The pyrene functionalized PANI which was precipitated from methanol solvent was soluble and well dispersed in polar solvents like $N, N$-dimethylformamide, dimethyl sulfoxide, $N$-methyl pyrrolidone and maximum solubility was found to be $2 \% \mathrm{w} / \mathrm{v}$. When the pyrene functionalized PANI in $N, N$-dimethylformamide solvent was centrifuged at 2000 rpm, the solution was clear without any sediment on the wall of sample tube, indicating that pyrene functionalized PANI is soluble and well dispersed in $N, N$-dimethylformamide solvent. The pyrene moieties present in the polyaniline backbone may provide space for the penetration of solvent molecules inside the chain and induces solubility of polymer in polar solvents. The solubility of pyrene functionalized PANI in solvents like dichloromethane, chloroform, and tetrahydrofuran was also observed. This result is in accordance with the solubility of polyaniline containing long chain dopants, such as dodecylbenzenesulfonic acid. ${ }^{31}$

True solutions of conducting polymers not only do not exist, but generally they cannot exist; the reason being that the surface tension for the conducting polymers is far higher than that of any of the available solvents. To form true solutions, the surface tension of the solvent must be similar to that of the solute. Water has a surface tension of $72.9 \mathrm{mN} /$ $\mathrm{m}$, the highest known value for a solvent, but still far below the $150 \mathrm{mN} / \mathrm{m}$ observed in PANI. ${ }^{32}$ Since PANI cannot exist in true solution, the well-dispersed form of PANI can be considered as solution in the place of true solution.

The Figure 3 shows the photoluminescence (PL) spectrum of the pyrene-PANI in meta-cresol solvent. The PL spectrum of the pyrene-PANI reveals that the emission lies in blue region, with the peak of $475 \mathrm{~nm}$ and the excitation wave- 


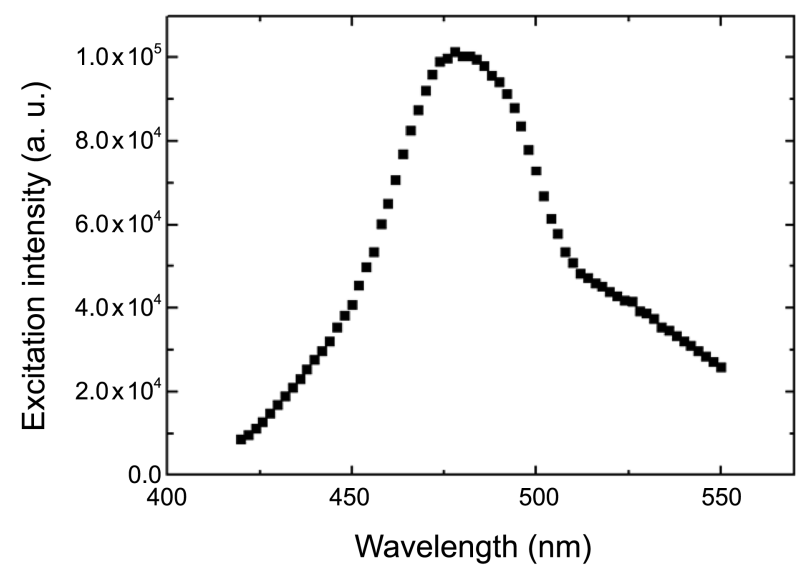

Figure 3. Photoluminescence spectrum of pyrene functionalized PANI.

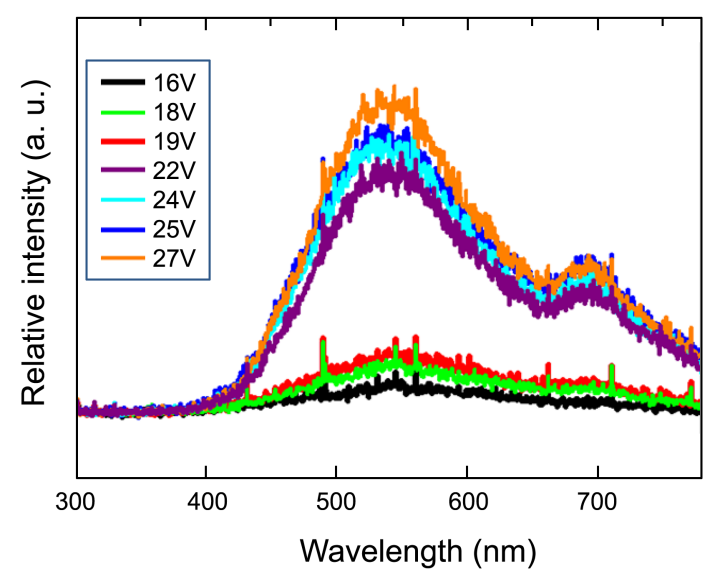

Figure 4. Electroluminescence spectra of pyrene functionalized PANI with various input voltages.

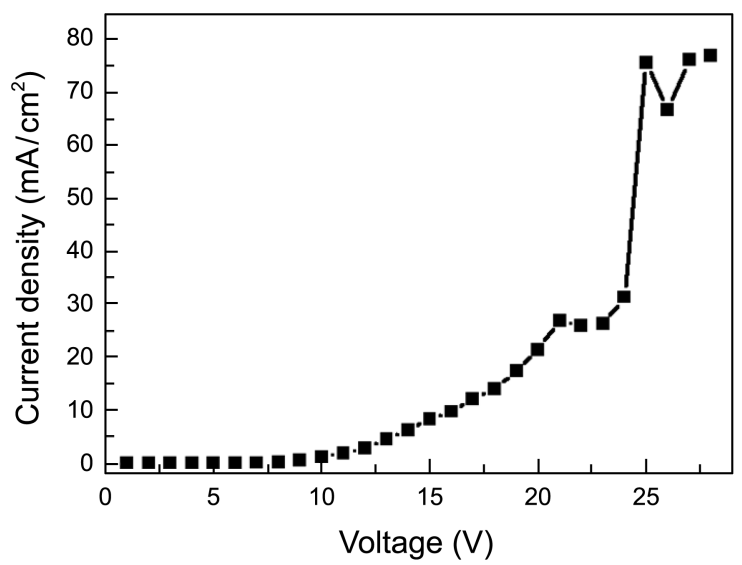

Figure 5. I-V characteristics of pyrene funtionalized polyaniline as emissive layer.

length of $355 \mathrm{~nm}$. The Figure 4 shows the electroluminescence (EL) spectra of pyrene functionalized polyaniline molecules with various input voltages. The spectra show that the pyrene functionalized PANI is stable at higher voltage like 22 to $27 \mathrm{~V}$. The intensity increased slightly when the input voltage was increased from 16 to $19 \mathrm{~V}$. When the input voltage was increased to $22-27 \mathrm{~V}$, the intensity increased drastically. The detected EL deviates from the PL spectrum. In the EL the emission is significantly shifted towards (orange light emission) longer wavelength around $525 \mathrm{~nm}$. The reason may be an interaction of the pyrene moieties with polyaniline chain. This so-called "solvent shift" leads to a shift of the emission wavelength of each pyrene molecule depending on its environment. ${ }^{33} \mathrm{We}$ suppose that different chain lengths are probably responsible for the shoulder shape of the electroluminescence spectrum. Thus, one can explain the wide band of emitted frequencies. To demonstrate the potential application of this pyrene functionalized polyaniline in organic polymer electronic applications, organic light emitting diode was prepared using pyrene containing polyaniline as emissive layer. ${ }^{34}$ The device structure is as follows ITO/PEDOT:PSS $(25 \mathrm{~nm}) /$ pyrenePANI $(55 \mathrm{~nm}) / \mathrm{LiF}(1 \mathrm{~nm}) / \mathrm{Al}(150 \mathrm{~nm})$.

Figure 5 shows that the turn-on voltage of the device is $15 \mathrm{~V}$. The higher the turn-on voltage the lower the device efficiency. We believe that the lower efficiency of the device may be due to the interactions between the polyaniline chain and pyrene rings, or it may be due to the insufficient amount of pyrene on the polyaniline chain. In this regard, we are planning to improve the device efficiency by increasing the pyrene amount in polyaniline chain, and also by using the oligomeric aniline in presence of polyaniline chain.

\section{Conclusions}

In summary, reported here is a new class of solution processable amorphous material based on pyrene and polyaniline with many attractive properties for potential application in polymer/organic light emitting diodes technology including ease of synthesis, low cost, high glass transition temperature, good film-forming and light-emitting properties. The preliminary PL and EL properties of the material demonstrate the utility of this material as emitters in polymer light emitting diodes. Future work in this area will explore the pyrene functionalized with other conductive polymers to give reasonable turn-on voltage and for other applications like organic photovoltaics, and organic thin-film transistors.

Acknowledgments. This research was supported by GRRC program of Gyeonggi Province, 2008-0169, low dimensional nanoprobe synthesis for the application of chem-biosensors.

\section{References}

1. Nalwa, H. S. Handbook of Organic Conductive Molecules and Polymers; John Wiley \& Sons: Chichester, 1997.

2. Wei, Y.; Focke, W. W.; Wnek, G. E.; Ray, A.; MacDiarmid, A. G. J. Phys. Chem. 1989, 93, 495.

3. D’Aprano, G.; Leclerc, M.; Zotti, G.; Schiavon, G. Chem. Mater. 1995, 7, 33.

4. Tang, C. W.; Van Slyke, S. A. Appl. Phys. Lett. 1987, 51, 913.

5. Tang, C. W.; Van Slyke, S. A.; Chen, C. H. J. Appl. Phys. 1989, 65,3610 .

6. Chen, C. H.; Shi, J.; Tang, C. W. Macromol. Symp. 1997, 125, 1.

7. Antoniadis, H.; Inbasekaran, M.; Woo, E. P. Appl. Phys. Lett. 1998, 73, 3055 . 
8. Hoskawa, C.; Higashi, H.; Nakamura, H.; Kusumoto, T. Appl. Phys. Lett. 1995, 67, 3853.

9. Noda, T.; Ogawa, H. H.; Shirota, Y. Adv. Mater. 1999, 11, 283.

10. Chen, C. H.; Tang, C. W.; Shi, J.; Klubek, K. P. Macromol. Symp. 1997, 125, 49.

11. Shen, Z.; Burrows, P. E.; Bulovic, V.; Forrest, S. R.; Thompson, M. E. Science 1997, 276, 2009.

12. Xie, Z. Y.; Huang, J. S.; Li, C. N.; Liu, S. Y.; Wang, Y.; Li, Y. Q.; Shen, J. C. Appl. Phys. Lett. 1999, 74, 641.

13. Kido, J.; Lizumi, Y. Appl. Phys. Lett. 1998, 73, 2721.

14. Van Slyke, S. A.; Chen, C. H.; Tang, C. W. Appl. Phys. Lett. 1996, $69,2160$.

15. Shi, J.; Tang, C. W. Appl. Phys. Lett. 1997, 70, 1665.

16. Tang, C. W.; Van Slyke, S. A.; Chen, C. H. J. Appl. Phys. 1989, 65,3610 .

17. Baldo, M. A.; O’Brien, D. F.; You, Y.; Shoustikov, A.; Sibley, S.; Thompson, M. E.; Forrest, S. R. Nature 1998, 359, 151.

18. Burrows, P. E.; Forrest, S. R. Appl. Phys. Lett. 2000, 76, 2493.

19. Tang, C. W.; Van Slyke, S. A. Appl. Phys. Lett. 1987, 51, 913.

20. Burroughes, J. H.; Bradley, D. D. C.; Brown, A. R.; Marks, R. N.; Mackay, K.; Friend, R. H.; Burn, P. L.; Holmes, A. B. Nature 1990, 347, 539

21. Hung, L. S.; Chen, C. H. Mater. Sci. Eng R 2002, 39, 143.

22. Akcelrud, L. Prog. Polym. Sci. 2003, $28,875$.

23. Hatwar, T. K. In The 5th International Conference on Electroluminescence of Molecular Materials and Related Phenomena (ICEL-5); Phoenix, 2005.
24. Pardo, D. A.; Jabbour, G. E.; Peyghambarian, N. Adv. Mater. 2000, $12,1249$.

25. de Gans, B.; Duineveld, P. C.; Schubert, U. S. Adv. Mater. 2004, $16,203$.

26. Palaniappan, S.; John, A.; Amarnath, C. A.; Rao, V. J. J. Mol. Cat. A: Chemical 2004, 218, 47.

27. Hwang, G. W.; Wu, K. Y.; Hua, M. Y.; Lee, H. T.; Chen, S. A. Synth. Met. 1998, 92, 39.

28. Amarnath, C. A.; Kim, J.; Kim, K.; Choi, J.; Sohn, D. Polymer $\mathbf{2 0 0 8}, 49,432$

29. Rao, P. S.; Satyanarayana, D. N.; Palaniappan, S. Macromolecules 2002, 35, 4988.

30. Lo, M. Y.; Zhen, C.; Lauters, M.; Jabbour, G. E.; Sellinger, A. $J$. Am. Chem. Soc. 2007, 129, 5808.

31. Shreepathi, S.; Holze, R. Chem. Mater. 2005, 17, 4078.

32. Wessling, B. Chem. Innov. 2001, 35.

33. Avnir, D.; Kaufman, V. R.; Reisfeld, R. J. Non-Cryst Solids 1985, $74,395$.

34. In a typical experiment, ITO coated glass substrates were ultrasonically cleaned in sequential steps using acetone, methanol, and isopropanol. Poly(ethylenedioxythiophene)-polystyrene sulfonic acid (PEDOT-PSS, $2.5 \%$ in water) was spin coated, followed by baking at $100{ }^{\circ} \mathrm{C}$ under nitrogen for 30 min to provide a $25 \mathrm{~nm}$ layer. Active material was spin-coated from chloroform (pyrene functionalized polyaniline) $20 \mathrm{mg} / \mathrm{mL}$ to yield a thickness of 55 $\mathrm{nm}$. The substrate was then loaded into a vacuum chamber and $\mathrm{LiF}(1 \mathrm{~nm}), \mathrm{Al}(150 \mathrm{~nm})$ were deposited. 